\title{
UNIFORM ADIABATIC LIMIT OF BENNEY TYPE SYSTEMS
}

\author{
ADÁN J. CORCHO \\ Instituto de Matemática, Universidade Federal do Rio de Janeiro. \\ Centro de Tecnologia - Bloco C. Cidade Universitária. \\ Ilha do Fundão 21941-909. Rio de Janeiro - RJ, Brazil. \\ JUAN C. CORDERO \\ Departamento de Matemáticas y Estadística \\ Universidad Nacional de Colombia \\ Campus La Nubia - Manizales
}

\begin{abstract}
In this paper we show that solutions of the cubic nonlinear Schrödinger equation are asymptotic limit of solutions to the Benney system. Due to the special characteristic of the one-dimensional transport equation same result is obtained for solutions of the onedimensional Zakharov and 1d-Zakharov-Rubenchik systems. Convergence is reached in the topology $L^{2}(\mathbb{R}) \times L^{2}(\mathbb{R})$ and with an approximation in the energy space $H^{1}(\mathbb{R}) \times L^{2}(\mathbb{R})$. In the case of the Zakharov system this is achieved without the condition $\partial_{t} n(x, 0) \in \dot{H}^{-1}(\mathbb{R})$ for the wave component, improving previous results.
\end{abstract}

\section{INTRODUCTION}

We consider a family of one-dimensional nonlinear dispersive systems, given by the following coupling equations:

$$
\left\{\begin{array}{l}
i \partial_{t} u+\partial_{x}^{2} u=\left(\tau|u|^{2}+\alpha v+\alpha^{\prime} z\right) u, \quad(x, t) \in \mathbb{R} \times \mathbb{R}^{+}, \\
\varepsilon \partial_{t} v+\lambda \partial_{x} v=\beta \partial_{x}|u|^{2}, \\
\varepsilon \partial_{t} z+\lambda^{\prime} \partial_{x} z=\beta^{\prime} \partial_{x}|u|^{2},
\end{array}\right.
$$

where $u$ is a complex-valued function, $v$ and $z$ are real-valued functions, the physical parameters $\tau, \alpha, \alpha^{\prime}$, $\lambda, \lambda^{\prime}, \beta, \beta^{\prime}$ are real numbers, and $0<\varepsilon<1$. This model governs, on certain parameter regimes, the dynamics of many physical phenomena and it is in the "neighborhood" of some other important models of the mathematical-physics; for example, the Zakharov system, the Davey-Stewartson system and the nonlinear Schrödinger equation. We give further information about well-posedness concerning System (1.1) in Section 6.

E-mail addresses: adan@im.ufrj.br, jccorderoc@unal.edu.co.

Date: June 25, 2020.

2010 Mathematics Subject Classification. Primary 35Q55, 35Q60; Secondary 35B65.

Key words and phrases. Perturbed Nonlinear Schrödinger Equation, Cauchy Problem, Asymptotic behavior.

A. J. Corcho was partially supported by CAPES and CNPq (307761/2016-9), Brazil.

J. C. Cordero was partially supported by the Departamento de Matemáticas y Estadística, Universidad Nacional de Colombia, Sede Manizales. 
The family (1.1) contains, for instance, some cases of the non resonant dynamics of small amplitude Alfven waves propagating in a plasma [10, 23], modeled by the coupled equations:

$$
\left\{\begin{array}{l}
i \partial_{t} u+\partial_{x}^{2} u=k\left(c|u|^{2}-\frac{1}{2} a \rho+\varphi\right) u \\
\varepsilon \partial_{t} \rho+\partial_{x}(\varphi-a \rho)=-k \partial_{x}|u|^{2} \\
\varepsilon \partial_{t} \varphi+\partial_{x}(b \rho-a \varphi)=\frac{1}{2} k \partial_{x}|u|^{2}
\end{array}\right.
$$

where we have taken the frequency $\omega$ equal to 1 , on the expanded flat wave front to generate $u$. This model is known as 1d-Zakharov-Rubenchik type system, which in the case $b>0$ and $b-a^{2} \neq 0$, using the transformation (see [19])

$$
\rho=\psi_{1}+\psi_{2}, \quad \varphi=\sqrt{b}\left(\psi_{1}-\psi_{2}\right)
$$

can be rewritten as

$$
\left\{\begin{array}{l}
i \partial_{t} u+\partial_{x}^{2} u=\left(c|u|^{2}-\left(\sqrt{b}+\frac{a}{2}\right) \psi_{2}+\left(\sqrt{b}-\frac{a}{2}\right) \psi_{1}\right) u \\
\varepsilon \partial_{t} \psi_{1}+(\sqrt{b}-a) \partial_{x} \psi_{1}=\frac{1}{2}\left(-1+\frac{a}{2 \sqrt{b}}\right) \partial_{x}|u|^{2} \\
\varepsilon \partial_{t} \psi_{2}-(a+\sqrt{b}) \partial_{x} \psi_{2}=\frac{1}{2}\left(-1-\frac{a}{2 \sqrt{b}}\right) \partial_{x}|u|^{2}
\end{array}\right.
$$

Another system included in the family (1.1) is the 1d-Zakharov system describing Langmuir turbulence [35], given by

$$
\left\{\begin{array}{l}
i \partial_{t} u+\partial_{x}^{2} u=n u, \\
\varepsilon^{2} \partial_{t}^{2} n-\partial_{x}^{2} n=\partial_{x}^{2}|u|^{2},
\end{array}\right.
$$

where $0<\varepsilon=k / c_{s}<1, k$ is a positive parameter and $c_{s}$ the ionic sound speed. It can be set in the form of (1.1) because we can write the wave equation of this system as

$$
\left(\varepsilon \partial_{t}-\partial_{x}\right)\left(\varepsilon \partial_{t}+\partial_{x}\right) n=\partial_{x}^{2}|u|
$$

and we make

$$
\left(\varepsilon \partial_{t} \pm \partial_{x}\right) n=\partial_{x} n_{\mp}
$$

to consider the two traveling wave profiles. Then we have

$$
\left\{\begin{array}{l}
i \partial_{t} u+\partial_{x}^{2} u=\frac{1}{2}\left(n_{-}-n_{+}\right) u \\
\varepsilon \partial_{t} n_{+}+\partial_{x} n_{+}=\partial_{x}|u|^{2} \\
\varepsilon \partial_{t} n_{-}-\partial_{x} n_{-}=\partial_{x}|u|^{2}
\end{array}\right.
$$

with

$$
\frac{1}{2}\left(n_{-}-n_{+}\right)=n
$$

In [15] a similar change of variables was performed for the Zakharov system, with complex positive and negative frequency parts for the wave equation, that is

$$
n_{ \pm}=n \pm i \omega^{-1} \partial_{t} n, \quad \omega=(-\Delta)^{1 / 2} .
$$

This allowed them to implement the Bourgain method to prove well posedness for the associated Cauchy problem in a wide class of Sobolev regularity for initial data.

From the physical point of view, the models (1.2) and (1.5) are magneto-hydrodynamics type systems in plasma physics [29, 17], however (1.2) is known as the Benney-Roskes system in the context of gravitational water waves [8].

A simpler model in the study of a general theory of water waves interactions in a nonlinear medium $[6,7]$, is the Benney system

$$
\left\{\begin{array}{l}
i \partial_{t} u+\partial_{x}^{2} u=\alpha u v, \\
\varepsilon \partial_{t} v+\lambda \partial_{x} v=\beta \partial_{x}|u|^{2},
\end{array}\right.
$$


where $|\lambda|=1$.

Before summarize some useful preliminary results we fix some notations. For a fixed positive time $T$, we are going to write

$$
\|f\|_{L_{T}^{\infty}}:=\sup _{t \in[0, T]}|f(t)|, \quad\|f\|_{L_{t}^{\infty}}:=\sup _{t \geq 0}|f(t)|
$$

and the symbol $\|\cdot\|_{L_{t}^{p} L_{x}^{q}}$ or $\|\cdot\|_{L_{T}^{p} L_{x}^{q}}$ will indicate the usual norm of a mixed space for $t \in \mathbb{R}^{+}$or $t \in[0, T]$, respectively.

We are interested in initial data belonging to $H^{s}$, which denotes the classical $L^{2}$-Sobolev space and also will be used the mixed norm of $L^{\infty}\left([0, T] ; H^{s}(\mathbb{R})\right)$, defined by

$$
\|f\|_{L_{T}^{\infty} H_{x}^{s}}:=\sup _{0 \leq t \leq T}\|f(\cdot, t)\|_{H^{s}} .
$$

Well-posedness for the Cauchy problem associated to the above models in the $H^{s}\left(\mathbb{R}^{d}\right)$ spaces, as well as other important properties of the dynamics of the solutions, have been extensively considered for many authors.

Regarding the system (1.2) there are a few works but this one started to get attention in the last years, see for instance $[23,24,19]$. As far as we know the best local and global well-posedness for (1.2) was established in [19] and in dimensions $d=2,3$ we refer to the works [28, 12, 21, 13] for recent advances concerning existence of solutions, asymptotic behavior, instability of standing waves and blow-up solutions.

About the existence of global solutions for (1.5) there are global weak solutions for initial data $\left(u_{0}, n_{0}, n_{1}\right) \in H^{1} \times L^{2} \times H^{-1}$ (with $\left.n_{1}(x)=\partial_{t} n(x, 0)\right)$ and smooth solutions

$$
u \in L^{\infty}\left([0, T] ; H^{m}\right), \quad n \in L^{\infty}\left([0, T] ; H^{m-1}\right),
$$

for any time $T>0$ and for initial data in $H^{m} \times H^{m-1} \times H^{m-2}$ with $m \geq 3$ (see [31]). Also, when (1.5) is written as in (3.10), the solutions $\left(u_{\varepsilon}, n_{\varepsilon}, v_{\varepsilon}\right) \in H^{3} \times H^{2} \times H^{1}$ associated to the bounded family of initial data are bounded uniformly respect to $\varepsilon$ in $H^{1} \times L^{2} \times L^{2}$. This fact was used in [1] to prove the weak convergence to the cubic nonlinear Schrödinger (cubic-NLS) equation. A local and global theory in all dimensions for the system (1.5) was established in [15], and improvements of this in the two dimensional case were established later in [3] and in one dimensional case below energy space in $[26,27]$.

On the other hand, in [30] it was proven the convergence of solutions of the $2 d$ and $3 d$ Zakharov system to the corresponding solutions of the cubic-NLS equation in the subsonic limit $(\varepsilon \rightarrow 0)$, more exactly, they proved:

Theorem $1.1([30])$. Let $m \geq[d / 2]+3(d=2,3), \partial_{t} n_{\varepsilon}(x, 0)=\nabla w_{0 \varepsilon}$ and $T$ the time of existence of solutions (independent of $\varepsilon$ ). Assume that

$$
\begin{aligned}
& \left\|u_{0 \varepsilon}\right\|_{H^{m+1}}+\sqrt{\varepsilon}\left\|w_{0 \varepsilon}\right\|_{H^{m}}+\left\|n_{0 \varepsilon}\right\|_{H^{m}} \leq C, \\
& \frac{1}{\sqrt{\varepsilon}}\left\|\nabla\left(n_{0 \varepsilon}+\left|u_{0 \varepsilon}\right|^{2}\right)\right\|_{H^{m-1}}+\left\|\nabla w_{0 \varepsilon}\right\|_{H^{m-1}} \leq C,
\end{aligned}
$$

and

$$
\lim _{\varepsilon \rightarrow 0}\left\|u_{0 \varepsilon}-u_{0}\right\|_{H^{m+1}}=0
$$

Then, we have

$$
\begin{aligned}
& n_{\varepsilon}+\left|u_{\varepsilon}\right|^{2} \rightarrow 0 \quad \text { in } \quad \mathcal{C}\left([0, T] \times \mathbb{R}^{d}\right), \\
& \nabla\left[n_{\varepsilon}+\left|u_{\varepsilon}\right|^{2}\right] \rightarrow 0 \quad \text { in } \quad \mathcal{C}\left([0, T] ; H^{m-2}\right), \\
& u_{\varepsilon}-u \rightarrow 0 \quad \text { in } \quad \mathcal{C}^{1}\left([0, T] \times \mathbb{R}^{d}\right) \cap \mathcal{C}^{1}\left([0, T] ; \mathcal{C}^{2}\right) .
\end{aligned}
$$


For solutions with small amplitude were obtained rates of this convergence in [1] for $d=1,2,3$. For example, when the dimension is $d=1$, we have:

Theorem 1.2 ([1]). Consider de Cauchy problem associated to the system (1.5). Let $m \geq 3$ and assume that initial data satisfies

$$
u_{0} \in H^{m+2}, n_{0} \in H^{m+1} \text { and } n_{1}=\partial_{t} n(x, 0) \in H^{m}\left(n_{1}=\partial_{x}^{2} w_{0} \text { with } \partial_{x} w_{0} \in L^{2}\right) .
$$

Then,

$$
\left\|u_{\varepsilon}(\cdot, t)-u(\cdot, t)\right\|_{H^{m}} \leq M(t)\left(\varepsilon+\sqrt{\varepsilon}\left\|n_{0}+\left|u_{0}\right|^{2}\right\|_{H^{m}}\right),
$$

and

$$
\left\|n_{\varepsilon}(\cdot, t)+|u(\cdot, t)|^{2}-\tilde{n}(\cdot, t / \varepsilon)\right\|_{H^{m-1}} \leq M(t)\left(\varepsilon+\sqrt{\varepsilon}\left\|n_{0}+\left|u_{0}\right|^{2}\right\|_{H^{m}}\right),
$$

for some function $M(t) \in L_{l o c}^{\infty}\left(\mathbb{R}^{+}\right)$, where

$$
i \partial_{t} u+\partial_{x}^{2} u=-|u|^{2} u, \quad u(x, 0)=u_{0}(x),
$$

and $\tilde{n}$ is a fitting corrector satisfying the wave equation

$$
\left\{\begin{array}{l}
\partial_{t t} \tilde{n}-\partial_{x}^{2} \tilde{n}=0, \\
\tilde{n}(x, 0)=n_{0}(x)+\left|u_{0}(x)\right|^{2}, \quad \partial_{t} \tilde{n}(x, 0)=0 .
\end{array}\right.
$$

In [25], it was found optimal rates for this convergence. Also, in [16] the convergence in this limit has been proved whenever the initial data are uniformly bound in $H^{5}$. After, in [22] the convergence was proven in the energy space, still maintaining the condition $n_{1} \in \dot{H}^{-1}$ for the solutions found in [9] in dimension $d=3$, and $\left|\varepsilon \nabla^{-1}\right| n_{1}$ decaying for high frequency.

Finally, for system (1.11) in the works $[4,5,11,15,18,33,34]$ the reader can find results about well-posedness, ill-posedness and existence/stability of solitary waves.

1.1. Goal and motivation. In the one-dimensional case all the systems above presented are globally well-posed in the natural energy space. Our interest here is to study the behavior of solutions of the Cauchy problem associated to (1.1), or equivalently of (1.11), in the energy space on any time interval $\Delta T:=[0, T]$ when $\varepsilon \rightarrow 0$. Formally, when $\varepsilon \rightarrow 0$ the system (1.1) decouples and the solutions $\left(u_{\varepsilon}, v_{\varepsilon}, w_{\varepsilon}\right)$ are reduced to satisfy

$$
\begin{cases}i \partial_{t} u+\partial_{x}^{2} u=\left(\tau+\frac{\alpha \beta}{\lambda}+\frac{\alpha^{\prime} \beta^{\prime}}{\lambda^{\prime}}\right) u|u|^{2}, & (x, t) \in \mathbb{R} \times(0, T], \\ v=\frac{\beta}{\lambda}|u|^{2}, & (x, t) \in \mathbb{R} \times[0, T], \\ z=\frac{\beta^{\prime}}{\lambda^{\prime}}|u|^{2}, & (x, t) \in \mathbb{R} \times[0, T],\end{cases}
$$

and in this case the limit is named adiabatic or subsonic because of the regime. The first component reaches to solve a cubic-NLS equation while the others components manage the quadratic nonlinearity.

In the case of (1.8) we have

$$
v=n_{+}=|u|^{2}, \quad z=n_{-}=-|u|^{2},
$$

with

$$
n=\frac{1}{2}\left(n_{-}-n_{+}\right)=-|u|^{2},
$$

and therefore the focusing cubic-NLS equation limit

$$
i \partial_{t} u+\partial_{x}^{2} u=-u|u|^{2} .
$$

Essentially we will have to deal with the system (1.11) because $v$ and $w$ are not coupled in (1.1), and in this case we have the limit system

$$
\begin{cases}i \partial_{t} u+\partial_{x}^{2} u=\frac{\alpha \beta}{\lambda} u|u|^{2}, & (x, t) \in \mathbb{R} \times(0, T], \\ v=\frac{\beta}{\lambda}|u|^{2}, & (x, t) \in \mathbb{R} \times[0, T],\end{cases}
$$


whose solution $u$ with initial data $u_{0}$ verifies

$$
u(x, t)=S(t) u_{0}-i \frac{\alpha \beta}{\lambda} \int_{0}^{t} S(t-s)|u(x, s)|^{2} u(x, s) d s,
$$

where

$$
S(t)=e^{i t \partial_{x}^{2}}
$$

denotes the unitary group associated to the linear Schrödinger equation.

Clearer evidence that this convergence is possible comes from the convergence of the traveling waves of the Benney system to those of equation (1.22). More precisely, for a given $c>0$ and $\alpha, \beta, \lambda$ satisfying

$$
\frac{\alpha \beta}{\lambda}<0
$$

the traveling waves

$$
\left\{\begin{array}{l}
u_{c, w, \varepsilon}(x, t)=e^{i w t} e^{\frac{i c}{2}(x-c t)} \sqrt{\frac{2(\varepsilon c-\lambda)}{\alpha \beta}} \sigma \operatorname{sech}(\sigma(x-c t)), \\
v_{c, w, \varepsilon}(x, t)=-\frac{2}{\alpha} \sigma^{2} \operatorname{sech}^{2}(\sigma(x-c t)),
\end{array}\right.
$$

with $0<\varepsilon<1 / c$ and $\sigma=\sqrt{w-c^{2} / 4}$ are solutions of the Benney system and, on the other hand, under condition (1.25) the family

$$
u_{c, w}(x, t)=e^{i w t} e^{\frac{i c}{2}(x-c t)} \sqrt{\frac{-2 \lambda}{\alpha \beta}} \sigma \operatorname{sech}(\sigma(x-c t)),
$$

describes solutions of (1.22). Then, by using the Lebesgue's dominated convergence theorem we can verify that

moreover

$$
\lim _{\varepsilon \rightarrow 0}\left\|u_{c, w, \varepsilon}-u_{c, w}\right\|_{L_{t}^{\infty} L_{x}^{2}}=0
$$

for all $\varepsilon>0$.

Traveling waves are global localized solutions of system (1.11) belonging to the energy space $H^{1} \times L^{2}$. However, for any initial data $\left(u_{0}, v_{0}\right) \in H^{1} \times L^{2}$ the corresponding solution $\left(u_{\varepsilon}, v_{\varepsilon}\right)$ of (1.11) is global in time, as we will described in Section 3.3 below. So, it's natural to ask whether the convergence $\left(u_{\varepsilon}, v_{\varepsilon}\right) \rightarrow\left(u, \frac{\beta}{\lambda}|u|^{2}\right)$ holds in $H^{1} \times L^{2}$ or at least in $L^{2} \times L^{2}$.

Remark 1.3. We highlight that the limit to the cubic-NLS showed in $[1,30,24]$ is in the sense of punctual convergence in time. Moreover, as in [25, 16], the technique used in the proof demanded more than four derivatives on the initial data. In [1] the authors also proved weak convergence by using compactness arguments on the energy space and in [24] it was used the theory of symmetric hyperbolic system to deal with the limit of (1.2) but the result of this work is weaker.

Our goal in this work is to improve, in some sense in one dimension, the known convergence results for these systems, taking as starting point the study of the convergence for solutions of the (1.11). The key point in (1.11) is that we will take advantage of the transport phenomenon in a single equation in order to find a rate for the convergence of solutions to the respective solution of (1.22) in the space $\mathcal{C}\left([0, T] ; L^{2} \times L^{2}\right)$. Then we replicate the same argument to get a rate for the convergence of solutions of the more general system (1.1) to the solution of the equation (1.18) in the space $\mathcal{C}\left([0, T] ; L^{2} \times L^{2} \times L^{2}\right)$. Consequently, we derive rates for the convergence of solutions of the systems (1.4) and (1.8), and also for system (1.5) in the respective topology induced by the energy space. In this sense our results are stronger.

Notice that in [30], the real-valued functions of the original system behaves like quadratic nonlinearity of the same system as $\varepsilon$ tends to 0 , but this is not met in the limit. We also can prove that this is really achieved too, with less regular initial data, as long as the solutions stay uniformly bounded 
respect to $\varepsilon$. Therefore, our results are a significant improvement, which will be established in the next section.

\section{MAin Results}

It will be important to distinguish solutions corresponding to different values of $\varepsilon$ for system (1.1), which justifies the notation $u_{\varepsilon}=u_{\varepsilon}(x, t), v_{\varepsilon}=v_{\varepsilon}(x, t)$ and $z_{\varepsilon}=z_{\varepsilon}(x, t)$ whenever this is necessary.

Theorem 2.1. Let $T>0$ be given. Suppose that the solutions $\left(u_{\varepsilon}, v_{\varepsilon}, z_{\varepsilon}\right)$ of system (1.1) corresponding to a family of initial data $\left\{\left(u_{0 \varepsilon}, v_{0 \varepsilon}, z_{0 \varepsilon}\right)\right\}_{0<\varepsilon<1} \in H^{1} \times L^{2} \times L^{2}$, satisfy

$$
\sup _{0<\varepsilon<1}\left(\left\|u_{\varepsilon}\right\|_{L_{T}^{\infty} H_{x}^{1}}+\left\|v_{\varepsilon}\right\|_{L_{T}^{\infty} L_{x}^{2}}+\left\|z_{\varepsilon}\right\|_{L_{T}^{\infty} L_{x}^{2}}\right)<\infty
$$

and

$$
\left|u_{\varepsilon}\right| \leq \psi \quad \text { a.e, }
$$

for some $\psi \in L^{\infty}\left([0, T] ; L^{\infty} \cap L^{2}\right)$, and let $u$ the solution of the cubic nonlinear Schrödinger equation in (1.18) with data $u_{0} \in H^{1}$. Then,

$$
\lim _{\varepsilon \rightarrow 0}\left(\left\|u_{\varepsilon}-u\right\|_{L_{T}^{\infty} L_{x}^{2}}+\left\|v_{\varepsilon}-\frac{\beta}{\lambda}|u|^{2}\right\|_{L_{T}^{\infty} L_{x}^{2}}+\left\|z_{\varepsilon}-\frac{\beta^{\prime}}{\lambda^{\prime}}|u|^{2}\right\|_{L_{T}^{\infty} L_{x}^{2}}\right)=0
$$

whenever the family of initial data satisfies

$$
\lim _{\varepsilon \rightarrow 0}\left(\left\|u_{0 \varepsilon}-u_{0}\right\|_{H^{1}}+\left\|v_{0 \varepsilon}-\frac{\beta}{\lambda}\left|u_{0}\right|^{2}\right\|_{L^{2}}+\left\|z_{0 \varepsilon}-\frac{\beta^{\prime}}{\lambda^{\prime}}\left|u_{0}\right|^{2}\right\|_{L^{2}}\right)=0
$$

Theorem 2.2. Let $T>0$ be given and consider $\frac{\alpha \lambda}{\beta}<0$. Suppose that $\left\{\left(u_{0 \varepsilon}, v_{0 \varepsilon}\right)\right\}_{0<\varepsilon<1}$ is a family of data in the space $H^{1} \times L^{2}$ such that

$$
\sup _{0<\varepsilon<1}\left(\left\|u_{0 \varepsilon}\right\|_{H^{1}}+\left\|v_{0 \varepsilon}\right\|_{L^{2}}\right)<\infty
$$

and

$$
\lim _{\varepsilon \rightarrow 0}\left\|v_{0 \varepsilon}-\frac{\beta}{\lambda}\left|u_{0 \varepsilon}\right|^{2}\right\|_{L^{2}}=0
$$

Then, the corresponding solutions $\left(u_{\varepsilon}, v_{\varepsilon}\right)$ of the Cauchy problem associated to the $(1.11)$ in $H^{1} \times L^{2}$ provided in [15] satisfies

$$
\left\|v_{\varepsilon}-\frac{\beta}{\lambda}\left|u_{\epsilon}\right|^{2}\right\|_{L_{T}^{\infty} L_{x}^{2}}=O(\varepsilon)
$$

and

$$
\left\|\int_{0}^{t} S(t-s)\left[u_{\varepsilon} v_{\varepsilon}-\frac{\beta}{\lambda} u_{\varepsilon}\left|u_{\varepsilon}\right|^{2}\right](x, s) d s\right\|_{L_{T}^{\infty} L_{x}^{2}} \lesssim T^{3 / 4} O(\varepsilon) .
$$

Furthermore, the same is true in the case $\frac{\alpha \lambda}{\beta}>0$ under the extra hypothesis $\frac{\beta}{\lambda^{2}}=O\left(\epsilon^{3}\right)$.

Corollary 2.3. Assume the hypotheses in Theorem 2.2 in the case $\frac{\alpha \lambda}{\beta}<0$. Suppose further that

$$
\left|u_{\varepsilon}\right| \leq \psi \quad \text { a.e }
$$

for some function $\psi \in L^{\infty}\left([0, T] ; L^{\infty} \cap L^{2}\right)$. If $u$ is the solution of the cubic nonlinear Schrödinger equation (1.22) with initial data $u_{0} \in H^{1}$, then

$$
\lim _{\varepsilon \rightarrow 0}\left\|v_{\varepsilon}-\frac{\beta}{\lambda}|u|^{2}\right\|_{L_{T}^{\infty} L_{x}^{2}}=0
$$

and

$$
\lim _{\varepsilon \rightarrow 0}\left\|u_{\varepsilon}-u\right\|_{L_{T}^{\infty} L_{x}^{2}}=0
$$

whenever $\left\|u_{0 \varepsilon}-u_{0}\right\|_{L^{2}} \rightarrow 0$. 
Theorem 2.4. Consider the Cauchy problem associated to (1.11) with $\frac{\alpha \lambda}{\beta}<0$. If $\left\{\left(u_{0 \varepsilon}, v_{0 \varepsilon}\right)\right\}_{0<\varepsilon<1}$ is a family of data in the space $H^{1} \times L^{2}$ such that

$$
\lim _{\varepsilon \rightarrow 0}\left(\left\|u_{0 \varepsilon}-u_{0}\right\|_{H^{1}}+\left\|v_{0 \varepsilon}-\frac{\beta}{\lambda}\left|u_{0}\right|^{2}\right\|_{H^{1}}\right)=0
$$

and $u$ is the solution of (1.22) with initial data $u_{0}$, then we have

$$
\left\|\partial_{x} \int_{0}^{t} S(t-s)\left[u_{\varepsilon} v_{\varepsilon}-\frac{\beta}{\lambda} u_{\varepsilon}\left|u_{\varepsilon}\right|^{2}\right](x, s) d s\right\|_{L_{T}^{\infty} L_{x}^{2}} \lesssim T^{3 / 4} O(\varepsilon) .
$$

Remark 2.5. It is important to note that:

(a) The inequalities (2.8) and (2.13) means, respectively, that

$$
\left\|u_{\varepsilon}-S(t) u_{0 \epsilon}+i \frac{\alpha \beta}{\lambda} \int_{0}^{t} S(t-s) u_{\varepsilon}\left|u_{\varepsilon}\right|^{2} d s\right\|_{L_{T}^{\infty} L_{x}^{2}} \lesssim T^{3 / 4} O(\varepsilon) .
$$

and

$$
\left\|\partial_{x}\left(u_{\varepsilon}-S(t) u_{0 \epsilon}+i \frac{\alpha \beta}{\lambda} \int_{0}^{t} S(t-s) u_{\varepsilon}\left|u_{\varepsilon}\right|^{2} d s\right)\right\|_{L_{T}^{\infty} L_{x}^{2}} \lesssim T^{3 / 4} O(\varepsilon) .
$$

(b) The sign $\frac{\alpha \lambda}{\beta}<0$ is because it enables to obtain bonded solutions respect to $\varepsilon$.

(c) For the 1d-Zakharov system we have removed the condition $n_{1} \in \dot{H}^{-1}$ whenever we have uniformly bounded solutions on the parameter $\varepsilon$. Also we do not impose decay for high frequency on initial data.

\section{Preliminary Results}

3.1. On the 1d-Zakharov-Rubenchik. As we commented in the introduction, the best known result about local well-posedness for the Zakharov-Rubenchik system (1.2) was established in [19]. The flux of this system preserves the following nonlinear quantities:

$$
\begin{gathered}
\mathcal{I}_{1}(t)=\int_{\mathbb{R}}|u(x, t)|^{2} d x \\
\mathcal{I}_{2}(t)=\frac{1}{2} \int_{\mathbb{R}}\left|\partial_{x} u(x, t)\right|^{2} d x+\frac{c k}{4} \int_{\mathbb{R}}|u(x, t)|^{4} d x+\frac{k}{2} \int_{\mathbb{R}}\left(\varphi-\frac{a}{2} \rho\right)(x, t)|u(x, t)|^{2} d x \\
+\frac{b}{4} \int_{\mathbb{R}}|\rho(x, t)|^{2} d x+\frac{1}{4} \int_{\mathbb{R}}|\varphi(x, t)|^{2} d x-\frac{b}{2} \int_{\mathbb{R}} \rho(x, t) \varphi(x, t) d x \\
\mathcal{I}_{3}(t)=\varepsilon \int_{\mathbb{R}} \rho(x, t) \varphi(x, t) d x+\frac{i \varepsilon}{2} \int_{\mathbb{R}}\left(u(x, t) \partial_{x} \bar{u}(x, t)-\bar{u}(x, t) \partial_{x} u(x, t)\right) d x
\end{gathered}
$$

and consequently

$$
\mathcal{I}_{4}(t)=\mathcal{I}_{2}(t)+\frac{b}{2 \varepsilon} \mathcal{I}_{3}(t)
$$

The conservation laws above yield the following global well-posedness:

Theorem 3.1 ([19]). The Cauchy problem associated to the system (1.4) is globally well posed for any initial data $\left(u_{0}, \psi_{10}, \psi_{20}\right)$ belonging to the spaces:

(a) $H^{s+1 / 2} \times H^{s} \times H^{s}$ with $s \geq 0$,

(b) $H^{1} \times L^{2} \times L^{2}$ whenever $b>a^{2}$.

Furthermore, in the energy space the solutions satisfied the uniform control

$$
\left\|\left(u(t), \psi_{1}(t), \psi_{2}(t)\right)\right\|_{H^{1} \times L^{2} \times L^{2}} \lesssim\left\|\left(u_{0}, \psi_{10}, \psi_{20}\right)\right\|_{H^{1} \times L^{2} \times L^{2}}^{2}+\left\|u_{0}\right\|_{L^{2}}^{6},
$$

for all $t \geq 0$. 
For more details on this matter we recommend [19, 23]. Also notice that if the initial data in the Zakahrov-Rubenchik system (depending on $\varepsilon$ ) satisfy the compatibility conditions (2.4) then the solutions are bounded uniformly.

3.2. On the 1d-Zakharov system. Concerning the existence of local solutions for the Cauchy problem associated to (1.5), a wide class of regularity was obtained in [15] for data

$$
\left(u_{0}, n_{0}, n_{1}\right) \in H^{s} \times H^{\kappa} \times H^{\kappa-1} \text {, with } n_{1}=n_{t}(0),
$$

in the case $\varepsilon=1$. We notice that the same theory holds for all $\varepsilon>0$ because of the re-scaling:

$$
u(x, t)=U\left(\varepsilon^{-1} x, \varepsilon^{-2} t\right) \text { and } n(x, t)=N\left(\varepsilon^{-1} x, \varepsilon^{-2} t\right),
$$

which transform the system (1.5) to

$$
\left\{\begin{array}{l}
i \partial_{r} U+\partial_{z}^{2} u=\varepsilon^{2} N U \\
\partial_{r}^{2} N-\partial_{z}^{2} N=\partial_{x}^{2}|U|^{2},
\end{array}\right.
$$

with $r=\varepsilon^{-2} t$ and $z=\varepsilon^{-1} x$. Indeed, system (3.6) is included in the local theory developed in [15], since bi-linear estimates using for the authors do not depend on the coefficients of nonlinear terms. More precisely, we have the following result:

Theorem 3.2 $([15])$. For any $\left(u_{0}, n_{0}, n_{1}\right) \in H^{s}(\mathbb{R}) \times H^{\kappa}(\mathbb{R}) \times H^{\kappa-1}$, with $s$ and $\kappa$ verifying the conditions:

$$
-\frac{1}{2}<s-\kappa \leq 1 \quad \text { and } \quad-\frac{1}{2} \leq \kappa \leq 2 s-\frac{1}{2},
$$

there exists a positive time $T=T\left(\left\|u_{0}\right\|_{H^{s}},\left\|n_{0}\right\|_{H^{\kappa}},\left\|n_{1}\right\|_{H^{\kappa-1}}\right)$ and a unique solution $(u(t, \cdot), v(t, \cdot))$ of the initial value problem (1.5) in the time interval $[0, T]$, satisfying

$$
\left(u, n, n_{t}\right) \in \mathcal{C}\left([0, T] ; H^{s}(\mathbb{R}) \times H^{\kappa}(\mathbb{R}) \times H^{\kappa-1}(\mathbb{R})\right) .
$$

Moreover, the map $\left(u_{0}, n_{0}, n_{1}\right) \longmapsto\left(u(\cdot, t), n(\cdot, t), n_{t}(\cdot, t)\right)$ is locally Lipschitz.

The Figure 1 shows the region $\mathcal{W}$ of the Sobolev indexes defined by conditions (3.7).

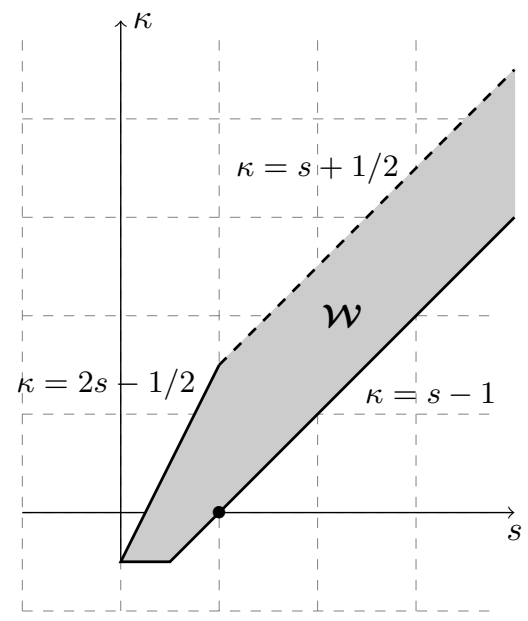

Figure 1. Local well-posedness regularity for (1.5) established in [15].

As we see, energy regularity $H^{1} \times L^{2} \times H^{-1}$ is covered in Theorem 3.2, so global well-posedness in this space is automatically provided by conservation laws:

$$
\mathcal{J}_{1}(t)=\int_{\mathbb{R}}|u(x, t)|^{2} d x
$$


and

$$
\mathcal{J}_{2}(t)=\int_{-\infty}^{+\infty}\left(\left|\partial_{x} u(x, t)\right|^{2}+n(x, t)|u(x, t)|^{2}+\frac{1}{2} n^{2}(x, t)+\frac{\varepsilon^{2}}{2}|v(x, t)|^{2}\right) d x,
$$

due to the Hamiltonian version of the Zakharov system (1.5):

$$
\left\{\begin{array}{l}
i \partial_{t} u+\partial_{x x} u=n u, \\
\partial_{t} n+\partial_{x} v=0, \\
\varepsilon^{2} \partial_{t} v+\partial_{x} n=-\partial_{x}|u|^{2},
\end{array}\right.
$$

where $v(x, t)=\partial_{x} w(x, t)$ and $\partial_{t} n(x, 0)=n_{1}(x)=-\partial_{x x} w_{0}$ for a certain function $w$.

We can notice that

$$
v=\partial_{x}^{-1} \partial_{t} n=\partial_{x}^{-1} n_{1} \quad \text { in } t=0,
$$

so $\mathcal{J}_{2}$ can be written only in terms of $u$ and $n$.

Finally, as solutions of (1.8) are solutions of (1.5), where

$$
\partial_{x} n_{\mp}=\varepsilon \partial_{t} n \pm \partial_{x} n,
$$

then

$$
\partial_{x} n_{\mp}=-\varepsilon \partial_{x} v \pm \partial_{x} n=\partial_{x}(-\varepsilon v \pm n)
$$

SO

$$
n_{\mp}=-\varepsilon v \pm n
$$

and

$$
\left\|n_{\mp}\right\|_{L^{2}} \leq \varepsilon\|v\|_{L^{2}}+\|n\|_{L^{2}} .
$$

This indicates a control of the components $n_{\mp}$ in function of the original variables $v$ and $n$, whence the solutions $\left(u_{\varepsilon}, n_{\varepsilon_{ \pm}}\right)$are uniformly bounded in $H^{1} \times L^{2}$. However, as we will see later, the system (1.1) has an intrinsically very good structure with three conserved quantities, so we do not need to assume $n_{1} \in \dot{H}^{-1}$ as in (3.10) to have a control of the solutions on $\varepsilon$.

3.3. On the Benney system. We will denote by $\mathcal{T}_{\varepsilon}(t)$ the family of translator operators associated to the free wave equation

$$
\left\{\begin{array}{l}
v_{t}+\frac{\lambda}{\varepsilon} v_{x}=0, \\
v(x, 0)=v_{0},
\end{array}\right.
$$

that is,

$$
\mathcal{T}_{\varepsilon}(t) v_{0}=v_{0}\left(x-\frac{\lambda}{\varepsilon} t\right)
$$

The most general theory concerning local well-posedness, known so far, in Sobolev spaces for the Cauchy problem associated to the Benney system (1.11) also was derived in [15], where the authors established local well-podness for initial data $\left(u_{0}, v_{0}\right) \in H^{s}(\mathbb{R}) \times H^{\kappa}(\mathbb{R})$ in the same region of regularity showed in Figure 1, with time of the existence $T$, depending on the norms $\left\|u_{0}\right\|_{H^{s}}$, and $\left\|v_{0}\right\|_{H^{\kappa}}$. Indeed, the results were obtained as a corollary of the proof of Theorem 3.2.

The solution for the system (1.11) with initial data $\left(u_{0_{\varepsilon}}, v_{0_{\varepsilon}}\right)$ satisfy the following integral equations:

$$
\left\{\begin{array}{l}
u_{\varepsilon}(x, t)=S(t) u_{0 \varepsilon}-i \alpha \int_{0}^{t} S(t-s) u_{\varepsilon}(x, s) v_{\varepsilon}(x, s) d s, \\
v_{\varepsilon}(x, t)=\mathcal{T}_{\varepsilon}(t) v_{0 \varepsilon}+\frac{\beta}{\varepsilon} \int_{0}^{t} \mathcal{T}_{\varepsilon}(t-s) \partial_{x}\left|u_{\varepsilon}(x, s)\right|^{2} d s .
\end{array}\right.
$$

The flow of the system (1.11) preserves the following nonlinear functional: 


$$
\begin{gathered}
\mathcal{M}_{\varepsilon}(t):=\int_{-\infty}^{+\infty}\left|u_{\varepsilon}(x, t)\right|^{2} d x=\mathcal{M}_{\varepsilon}(0) \quad(\text { mass }) \\
\mathcal{K}_{\varepsilon}(t):=\int_{-\infty}^{+\infty}\left(\left|v_{\varepsilon}(x, t)\right|^{2}+\frac{2 \beta}{\alpha \varepsilon} \operatorname{Im} u_{\varepsilon}(x, t) \partial_{x} \bar{u}_{\varepsilon}(x, t)\right) d x=\mathcal{K}_{\varepsilon}(0) \quad \text { (moment) }
\end{gathered}
$$

and

$$
\mathcal{E}_{\varepsilon}(t):=\int_{-\infty}^{+\infty}\left(\left|\partial_{x} u_{\varepsilon}(x, t)\right|^{2}+\alpha v_{\varepsilon}(x, t)\left|u_{\varepsilon}(x, t)\right|^{2}-\frac{\alpha \lambda}{2 \beta} v_{\varepsilon}^{2}(x, t)\right) d x=\mathcal{E}_{\varepsilon}(0), \quad \text { (energy) },
$$

for all $0 \leq t<T_{\varepsilon}^{*}$, where $T_{\varepsilon}^{*}$ is the maximal time of existence for the respective solution.

Our main goal is to study the asymptotic behavior for solutions of the Benney system in the topology of $\mathcal{C}\left([0, T] ; H^{1}(\mathbb{R})\right)$ for the component $u_{\varepsilon}$ and with an appropriated topology for the corresponding transport solution $v_{\varepsilon}$. However, if we expect a strong convergence result in $\mathcal{C}\left([0, T] ; L^{2}(\mathbb{R})\right)$ for solutions $v_{\varepsilon}$ it is natural to impose a compatibility condition on the initial data, like

$$
v_{0}=\frac{\beta}{\lambda}\left|u_{0}\right|^{2}
$$

or

$$
\lim _{\varepsilon \rightarrow 0}\left\|\lambda v_{0 \varepsilon}-\beta\left|u_{0 \varepsilon}\right|^{2}\right\|_{L^{2}}=0
$$

if the data vary with $\varepsilon$. For instance, if $u_{0 \varepsilon} \equiv 0$ for all $\varepsilon$ then

$$
u_{\varepsilon}(x, t) \equiv 0 \quad \text { and } \quad v_{\varepsilon}(x, t)=v_{0 \varepsilon}\left(x-\frac{\lambda}{\varepsilon} t\right),
$$

SO

$$
\left\|v_{\varepsilon}(x, t)\right\|_{L_{t}^{\infty} L_{x}^{2}}=\left\|v_{0 \varepsilon}\right\|_{L_{x}^{2}} .
$$

Hence, we do not have much chance of show convergence in the space $L_{T}^{\infty} H_{x}^{1} \times L_{T}^{\infty} L_{x}^{2}$ without assuming that $\left\|v_{0 \varepsilon}\right\|_{L_{x}^{2}} \rightarrow 0$ as $\varepsilon \rightarrow 0$. For non compatible initial data an initial layer phenomenon should appear.

3.4. Strichartz estimates. Finally, we recall some smoothing effects for the one-dimensional free Schrödinger group $S(t)$.

Lemma 3.3 (Strichartz estimates [14]). Let $\left(p_{1}, q_{1}\right)$ and $\left(p_{2}, q_{2}\right)$ be two pairs of admissible exponents for $S(t)$ in $\mathbb{R}$; that is, both satisfying the condition

$$
\frac{2}{p_{i}}=\frac{1}{2}-\frac{1}{q_{i}} \quad \text { and } \quad 2 \leq q_{i} \leq \infty \quad(i=1,2) .
$$

Then, for any $0<T \leq \infty$, we have

$$
\|S(t) f\|_{L_{T}^{p_{1}} L_{x}^{q_{1}}} \leq c\|f\|_{L^{2}(\mathbb{R})}
$$

as well as the non-homogeneous version

$$
\left\|\int_{0}^{t} S(t-s) g(\cdot, s) d s\right\|_{L_{T}^{p_{1}} L_{x}^{q_{1}}} \leq c\|g\|_{L_{T}^{p_{2}^{\prime}} L_{x}^{q_{2}^{\prime}}},
$$

where $1 / p_{2}+1 / p_{2}^{\prime}=1,1 / q_{2}+1 / q_{2}^{\prime}=1$. The constants in both inequalities are independent of $T$. 


\section{Acknowledgments}

A. J. Corcho would like to thank the support given by the Graduate Program in Mathematics of the Universidade Federal do Rio de Janeiro - UFRJ. J. C. Cordero would like to thank to the Instituto de Matemática at UFRJ and to the Instituto de Matemática Pura e Aplicada - IMPA for the support during the Postdoctoral Summer Program 2020, where part of this work was done. We would like to thank Hermano Frid for suggesting us to study the dynamics of the Benney system. We are also grateful to Felipe Linares for some useful comments on a previous version.

\section{EnERgy estimates AND WEAK CONVERGENCE}

In this section we present some estimates that will be useful to prove the statements of the main results. We describe the dynamic of the global solutions of (1.11) in the space $H^{1} \times L^{2}$ with respect to the parameter $\varepsilon$, when extra hypotheses are put on the initial data.

\subsection{A priori estimates for the Benney system.}

Lemma 4.1. If $\left\{\left(u_{0 \varepsilon}, v_{0 \varepsilon}\right)\right\}_{0<\varepsilon<1}$ is a family of data in the space $H^{1} \times L^{2}$ such that

$$
\sup _{0<\varepsilon<1}\left(\left\|u_{0 \varepsilon}\right\|_{H^{1}}+\left\|v_{0 \varepsilon}\right\|_{L^{2}}\right)<\infty,
$$

then the corresponding solutions $\left(u_{\varepsilon}, v_{\varepsilon}\right)$ of the IVP (1.11) in $H^{1} \times L^{2}$ provided by Theorem A satisfies

$$
\begin{aligned}
& \sup _{0<\varepsilon<1}\left(\left\|u_{\varepsilon}\right\|_{L_{t}^{\infty} H_{x}^{1}}+\left\|v_{\varepsilon}\right\|_{L_{t}^{\infty} L_{x}^{2}}\right)<\infty \quad \text { if } \quad \frac{\alpha \lambda}{\beta}<0, \\
& \left\|u_{\varepsilon}\right\|_{L_{t}^{\infty} H_{x}^{1}}+\left\|v_{\varepsilon}\right\|_{L_{t}^{\infty} L_{x}^{2}}=O(1 / \varepsilon) \quad \text { if } \quad \frac{\alpha \lambda}{\beta}>0 .
\end{aligned}
$$

Proof. We begin with the proof of (4.2). From (3.21) we have

$$
\begin{aligned}
\left\|\partial_{x} u_{\varepsilon}\right\|_{L^{2}}^{2}+\left|\frac{\alpha}{2 \beta}\right|\left\|v_{\varepsilon}\right\|_{L^{2}}^{2} & =\mathcal{E}_{\varepsilon}(0)-\alpha \int_{-\infty}^{+\infty} v_{\varepsilon}\left|u_{\varepsilon}\right|^{2} d x \\
& \leq \mathcal{E}_{\varepsilon}(0)+|\alpha|\left\|v_{\varepsilon}\right\|_{L^{2}}\left\|u_{\varepsilon}\right\|_{L^{4}}^{2} \\
& \leq \mathcal{E}_{\varepsilon}(0)+|\alpha|\left(\frac{1}{4|\beta|}\left\|v_{\varepsilon}\right\|_{L^{2}}^{2}+|\beta|\left\|u_{\varepsilon}\right\|_{L^{4}}^{4}\right) .
\end{aligned}
$$

So, by using Gagliardo-Nirenberg inequality and (3.19) we have

$$
\left\|\partial_{x} u_{\varepsilon}\right\|_{L^{2}}^{2}+\left|\frac{\alpha}{4 \beta}\right|\left\|v_{\varepsilon}\right\|_{L^{2}}^{2} \leq \mathcal{E}_{\varepsilon}(0)+c \mathcal{M}_{\varepsilon}^{3 / 2}(0)\left\|\partial_{x} u_{\varepsilon}\right\|_{L^{2}}
$$

that allows us to conclude

$$
\left\|\partial_{x} u_{\varepsilon}(\cdot, t)\right\|_{L^{2}}^{2}+\left\|v_{\varepsilon}(\cdot, t)\right\|_{L^{2}}^{2} \lesssim_{\alpha, \beta} \mathcal{E}_{\varepsilon}(0)+\mathcal{M}_{\varepsilon}^{3}(0), \quad \text { for all } t \geq 0 .
$$

Then, from (4.1) we deduce immediately (4.2).

Now we proceeds with the proof of (4.3). Again, using (3.21) and Gagliardo-Nirenberg inequality we obtain

$$
\begin{aligned}
\left\|\partial_{x} u_{\varepsilon}\right\|_{L^{2}}^{2} & =\mathcal{E}_{\varepsilon}(0)+\left|\frac{\alpha}{2 \beta}\right|\left\|v_{\varepsilon}\right\|_{L^{2}}^{2}-\alpha \int_{-\infty}^{+\infty} v_{\varepsilon}\left|u_{\varepsilon}\right|^{2} d x \\
& \leq \mathcal{E}_{\varepsilon}(0)+\left(\left|\frac{\alpha}{2 \beta}\right|+\left|\frac{\alpha}{2}\right|\right)\left\|v_{\varepsilon}\right\|_{L^{2}}^{2}+\left|\frac{\alpha}{2}\right|\left\|u_{\varepsilon}\right\|_{L^{4}}^{4} \\
& \leq \mathcal{E}_{\varepsilon}(0)+\left(\left|\frac{\alpha}{2 \beta}\right|+\left|\frac{\alpha}{2}\right|\right)\left\|v_{\varepsilon}\right\|_{L^{2}}^{2}+c\left|\frac{\alpha}{2}\right| \mathcal{M}_{\varepsilon}^{3 / 2}(0)\left\|\partial_{x} u_{\varepsilon}\right\|_{L^{2}} \\
& \leq \mathcal{E}_{\varepsilon}(0)+\left(\left|\frac{\alpha}{2 \beta}\right|+\left|\frac{\alpha}{2}\right|\right)\left\|v_{\varepsilon}\right\|_{L^{2}}^{2}+c_{\alpha, \beta} \mathcal{M}_{\varepsilon}^{3}+\frac{1}{4}\left\|\partial_{x} u_{\varepsilon}\right\|_{L^{2}}^{2} .
\end{aligned}
$$

On the other hand, from (3.20) we get

$$
\begin{aligned}
\left\|v_{\varepsilon}(\cdot, t)\right\|_{L^{2}}^{2} & \leq \mathcal{K}_{\varepsilon}(0)-\frac{2 \beta}{\alpha \varepsilon} \operatorname{Im} \int_{-\infty}^{+\infty} u_{\varepsilon} \partial_{x} \bar{u}_{\varepsilon} d x \\
& \leq \mathcal{K}_{\varepsilon}(0)+\left|\frac{2 \beta}{\alpha \varepsilon}\right| \mathcal{M}_{\varepsilon}^{1 / 2}(0)\left\|\partial_{x} u_{\varepsilon}\right\|_{L^{2}} \\
& \leq \mathcal{K}_{\varepsilon}(0)+\left|\frac{2 \beta^{2}}{\alpha^{2} \varepsilon^{2} \delta}\right| \mathcal{M}_{\varepsilon}(0)+\frac{\delta}{2}\left\|\partial_{x} u_{\varepsilon}\right\|_{L^{2}}^{2}
\end{aligned}
$$


for any positive $\delta$.

Then, taking a suitable $\delta=\delta(\alpha, \beta)$ and inserting (4.5) in (4.4) we have

$$
\left\|\partial_{x} u_{\varepsilon}(\cdot, t)\right\|_{L^{2}}^{2} \lesssim \alpha, \beta \mathcal{E}_{\varepsilon}(0)+\mathcal{K}_{\varepsilon}(0)+\mathcal{M}_{\varepsilon}(0)+\mathcal{M}_{\varepsilon}^{3}(0)
$$

Now we note that from $(4.1)$ we get $\mathcal{K}_{\varepsilon}(0)=O\left(\frac{1}{\varepsilon}\right), \mathcal{M}_{\varepsilon}(0)=O(1)$ and $\mathcal{E}_{\varepsilon}(0)=O(1)$. Thus, from (4.5) and (4.6) we obtain

$$
\left\|\partial_{x} u_{\varepsilon}(\cdot, t)\right\|_{L^{2}}^{2}+\left\|v_{\varepsilon}(\cdot, t)\right\|_{L^{2}}^{2}=O\left(1 / \varepsilon^{2}\right)
$$

which implies (4.3).

4.2. Weak limit for the Benney system. Now we state a weak convergence theorem for solutions $\left(u_{\varepsilon}, v_{\varepsilon}\right)$, namely

Theorem 4.2. Let $\left(u_{\varepsilon}, v_{\varepsilon}\right)$ be any solution of (1.11) with initial data satisfying the hypotheses of Lemma 4.1 and $\alpha \lambda / \beta<0$. There is $u \in L^{\infty}\left(\mathbb{R}_{+} ; H^{1}\right)$ such that $u_{\varepsilon} \rightarrow u$ almost everywhere in $(x, t) \in \mathbb{R} \times(0, T)$ as $\varepsilon$ go to 0 , and $\left(u_{\varepsilon}, v_{\varepsilon}\right)$ converges to $\left(u, \frac{\beta}{\lambda}|u|^{2}\right)$ in $L^{\infty}\left(\mathbb{R}_{+} ; H^{1}\right) \times L^{\infty}\left(\mathbb{R}_{+} ; L^{2}\right)$ weak star, where $u=u(x, t)$ is the unique solution of the nonlinear Schrödinger equation

$$
i \partial_{t} u+\partial_{x}^{2} u=\frac{\alpha \beta}{\lambda} u|u|^{2}
$$

with data $u(x, 0)=u_{0}(x) \in H^{1}$.

We only will do a sketch of the proof because these argument are well known. More details on this technicality can be review in [1, 12], even for higher dimensional models of Schrödinger type.

Proof. Because of the uniform bounds given by Lemma 4.1, we have a sequence $\left(u_{\varepsilon}, v_{\varepsilon}\right)$ and $(u, v)$ such that

$$
\left\{\begin{array}{lll}
u_{\varepsilon} \stackrel{*}{\rightarrow} u & \text { in } & L^{\infty}\left(\mathbb{R}_{+} ; H^{1}\right) \\
v_{\varepsilon} \stackrel{*}{\rightarrow} v & \text { in } & L^{\infty}\left(\mathbb{R}_{+} ; L^{2}\right) \\
\left|u_{\varepsilon}\right|^{2} \stackrel{*}{\rightarrow} \Gamma & \text { in } & L^{\infty}\left(\mathbb{R}_{+} ; L^{2}\right),
\end{array}\right.
$$

so

$$
\left\{\begin{array}{lll}
\partial_{x x} u_{\varepsilon} \stackrel{*}{*} \partial_{x x} u & \text { in } & L^{\infty}\left(\mathbb{R}_{+} ; H^{-1}\right) \\
\partial_{x} v_{\varepsilon} \stackrel{*}{*} v & \text { in } & L^{\infty}\left(\mathbb{R}_{+} ; H^{-1}\right) \\
\partial_{x}\left|u_{\varepsilon}\right|^{2} \stackrel{*}{\rightarrow} \partial_{x} \Gamma & \text { in } & L^{\infty}\left(\mathbb{R}_{+} ; H^{-1}\right)
\end{array}\right.
$$

As the map

$$
\begin{aligned}
H^{1} \times L^{2} & \rightarrow H^{-1} \\
(f, g) & \mapsto f g
\end{aligned}
$$

is continuous, one can assume that $u_{\varepsilon} v_{\varepsilon}$ has a weak ${ }^{*}$ limit in $L^{\infty}\left(\mathbb{R}_{+} ; H^{-1}\right)$, namely

$$
u_{\varepsilon} v_{\varepsilon} \stackrel{*}{\rightarrow} \Lambda \text { in } L^{\infty}\left(\mathbb{R}_{+} ; H^{-1}\right) .
$$

Then

$$
\left\{\begin{array}{lll}
\partial_{t} u_{\varepsilon} \stackrel{*}{\rightarrow} \partial_{t} u & \text { in } & L^{\infty}\left(\mathbb{R}_{+} ; H^{-1}\right) \\
\partial_{t} v_{\varepsilon} \stackrel{*}{\rightarrow} \partial_{t} v & \text { in } & L^{\infty}\left(\mathbb{R}_{+} ; H^{-1}\right)
\end{array}\right.
$$

and also

$$
\left\{\begin{array}{l}
i \partial_{t} u+\partial_{x}^{2} u=\alpha \Lambda \\
\lambda \partial_{x} v=\beta \partial_{x} \Gamma
\end{array}\right.
$$

in the distribution sense, in $L^{\infty}\left(\mathbb{R}_{+} ; H^{-1}\right)$. 
The proof of the result is finished when it is shown that

$$
\Gamma=|u|^{2} \quad \text { and } \quad \Lambda=\frac{\beta}{\lambda} u \Gamma .
$$

To check this we consider the interval $[0, T], \Omega \subset \mathbb{R}$ bounded, $B_{0}:=H^{1}(\Omega), B:=L^{4}(\Omega), B_{1}:=$ $H^{-1}(\Omega)$ and the restriction $\left.u_{\varepsilon}\right|_{\Omega}$. Next we use the Rellich-Kondrachov's theorem and the LionsAubin's theorem to have compact and continuous embeddings. Then some subsequence of $\left.u_{\varepsilon}\right|_{\Omega}$ (also labeled by $\varepsilon$ ) converges strongly to $\left.u\right|_{\Omega}$ in $L^{2}\left([0, T] ; L^{4}(\Omega)\right)$. Hence

$$
u_{\varepsilon} \underset{\varepsilon \rightarrow 0}{\longrightarrow} u \text { strongly in } L^{2}\left([0, T] ; L_{l o c}^{2}(\mathbb{R})\right),
$$

and thus,

$$
u_{\varepsilon} \underset{\varepsilon \rightarrow 0}{\longrightarrow} u \quad \text { a.e } \quad \text { in }(t, x) \in[0, T] \times \mathbb{R}
$$

and

$$
\left|u_{\varepsilon}\right|^{2} \underset{\varepsilon \rightarrow 0}{\longrightarrow}|u|^{2} \quad \text { a.e. } \quad \text { in }(t, x) \in[0, T] \times \mathbb{R}^{n} .
$$

Since $\left|u_{\varepsilon}\right|^{2} \in L^{\infty}\left((0, \infty) ; L^{2}(\mathbb{R})\right) \hookrightarrow L^{2}\left([0, T] ; L^{2}(\mathbb{R})\right)$ is bounded uniformly in $\varepsilon$, one gets

$$
\left|u_{\varepsilon}\right|^{2} \stackrel{*}{\rightarrow}|\psi|^{2} \quad \text { in } \quad L^{2}\left([0, T] ; L^{2}(\mathbb{R})\right)
$$

by reflexivity, so $\Gamma=|u|^{2}$.

The equality $\Lambda=\frac{\beta}{\lambda} u \Gamma$ follows by a standard argument.

\section{Proof of the Results}

\subsection{Proof of Theorem 2.2.}

Proof. As the transport solution in (3.18) is not easy to deal with, because of $\varepsilon \rightarrow 0$ and the spacial derivative in the nonlinearity, then one can rewrite the transport equation in (1.11) as

$$
\varepsilon \partial_{t}\left(v-\frac{\beta}{\lambda}|u|^{2}\right)+\lambda \partial_{x}\left(v-\frac{\beta}{\lambda}|u|^{2}\right)=-\frac{\varepsilon \beta}{\lambda} \partial_{t}|u|^{2},
$$

and with $w=v-\frac{\beta}{\lambda}|u|^{2}$ we have the solutions

$$
w_{\varepsilon}(x, t)=\mathcal{T}_{\varepsilon}(t) w_{0 \varepsilon}-\frac{\beta}{\lambda} \int_{0}^{t} \mathcal{T}_{\varepsilon}(t-s) \partial_{t}\left|u_{\varepsilon}(x, s)\right|^{2} d s .
$$

Notice that

$$
\mathcal{T}_{\varepsilon}(t) \partial_{t}=-\frac{\varepsilon}{\lambda} \partial_{t} \mathcal{T}_{\varepsilon}(t)
$$

consequently

$$
w_{\varepsilon}(x, t)=w_{0 \varepsilon}\left(x-\frac{\lambda}{\epsilon} t\right)+\frac{\varepsilon \beta}{\lambda^{2}}\left[1-\mathcal{T}_{\varepsilon}(t)\right]\left|u_{\varepsilon}(x, t)\right|^{2}
$$

where

$$
w_{0 \varepsilon}=v_{0 \varepsilon}-\frac{\beta}{\lambda}\left|u_{0 \varepsilon}\right|^{2} .
$$

As $\left\|u_{\varepsilon}(\cdot, t)\right\|_{L_{x}^{\infty}} \lesssim\left\|u_{\varepsilon}(\cdot, t)\right\|_{H^{1}}$ because $H^{1}(\mathbb{R}) \hookrightarrow C_{\infty}(\mathbb{R})$, then the first part of the theorem is now a immediate consequence of (5.3), the invariance by translation of $\|\cdot\|_{L^{p}(\mathbb{R})}$ and Lemma 4.1.

On the other hand,

then

$$
u_{\varepsilon} v_{\varepsilon}-\frac{\beta}{\lambda} u_{\epsilon}\left|u_{\varepsilon}\right|^{2}=u_{\varepsilon}\left(v_{\varepsilon}-\frac{\beta}{\lambda}\left|u_{\varepsilon}\right|^{2}\right),
$$

$$
\left\|u_{\varepsilon} v_{\varepsilon}-\frac{\beta}{\lambda} u_{\epsilon}\left|u_{\varepsilon}\right|^{2}\right\|_{L_{T}^{4 / 3} L_{x}^{1}} \lesssim T^{3 / 4}\left\|v_{\varepsilon}-\frac{\beta}{\lambda}\left|u_{\epsilon}\right|^{2}\right\|_{L_{T}^{\infty} L_{x}^{2}}
$$

follows by the Cauchy-Schwartz inequality and the uniform boundedness of $u_{\varepsilon}$ in the energy space again, due to the hypothesis. 
Now the proof is finished because of the smoothing effect:

$$
\left\|\int_{0}^{t} S(t-s)\left[u_{\varepsilon} v_{\varepsilon}-\frac{\beta}{\lambda} u_{\varepsilon}\left|u_{\varepsilon}\right|^{2}\right](x, s) d s\right\|_{L_{T}^{\infty} L_{x}^{2}} \lesssim\left\|u_{\varepsilon} v_{\varepsilon}-\frac{\beta}{\lambda} u_{\varepsilon}\left|u_{\varepsilon}\right|^{2}\right\|_{L_{T}^{4 / 3} L_{x}^{1}} \lesssim T^{3 / 4} O(\epsilon) .
$$

\subsection{Proof of Corollary 2.3.}

Proof. We have that

$$
\left\|u_{\varepsilon}-S(t) u_{0 \epsilon}+i \frac{\alpha \beta}{\lambda} \int_{0}^{t} S(t-s) u_{\varepsilon}\left|u_{\varepsilon}\right|^{2} d s\right\|_{L_{T}^{\infty} L_{x}^{2}} \lesssim T^{3 / 4} O(\varepsilon),
$$

so it is enough to verify that

$$
\left\|u-S(t) u_{0 \epsilon}+i \frac{\alpha \beta}{\lambda} \int_{0}^{t} S(t-s) u_{\varepsilon}\left|u_{\varepsilon}\right|^{2} d s\right\|_{L_{T}^{\infty} L_{x}^{2}} \longrightarrow 0
$$

as $\varepsilon \rightarrow 0$, and apply the triangular inequality. Here

$$
u(x, t)=S(t) u_{0}(x)-i \frac{\alpha \beta}{\lambda} \int_{0}^{t} S(t-s) u(x, s)|u(x, s)|^{2} d s,
$$

so it is only necessary to see for the nonlinear part.

We have

$$
\left.\left.\left|\int_{0}^{t} S(t-s) u_{\varepsilon}\right| u_{\varepsilon}\right|^{2} d s\right|^{2} \leq\left(\int_{0}^{t}\left|u_{\varepsilon}\right|\left|u_{\varepsilon}\right|^{2} d s\right)^{2} \leq\left(\int_{0}^{t}|\psi \| \psi|^{2} d s\right)^{2}
$$

and

$$
\begin{aligned}
\left(\int_{-\infty}^{+\infty}\left(\int_{0}^{t}|\psi||\psi|^{2} d s\right)^{2} d x\right)^{1 / 2} & \leq \int_{0}^{t}\left\|\left|\psi\left\|\left.\psi\right|^{2}\right\|_{L_{x}^{2}} d s\right.\right. \\
& \leq \int_{0}^{t}\|\psi\|_{L_{x}^{\infty}}^{2}\|\psi\|_{L_{x}^{2}} d s \\
& \leq T\|\psi\|_{L_{T}^{\infty} L_{x}^{\infty}}^{2}\|\psi\|_{L_{T}^{\infty} L_{x}^{2}} .
\end{aligned}
$$

As $u_{\varepsilon} \rightarrow u$ a.e, because of Theorem 4.2, the dominated convergence theorem give us

$$
\lim _{\varepsilon \rightarrow 0}\left\|\int_{0}^{t} S(t-s) u_{\varepsilon}\left|u_{\varepsilon}\right|^{2} d s\right\|_{L_{T}^{\infty} L_{x}^{2}}=\left\|\int_{0}^{t} S(t-s) u|u|^{2} d s\right\|_{L_{T}^{\infty} L_{x}^{2}} .
$$

Hence, we can conclude that $\lim _{\varepsilon \rightarrow 0}\left\|u_{\varepsilon}-u\right\|_{L_{T}^{\infty} L_{x}^{2}}=0$ and the convergence $\lim _{\varepsilon \rightarrow 0}\left\|v_{\varepsilon}-\frac{\beta}{\lambda}|u|^{2}\right\|_{L_{T}^{\infty} L_{x}^{2}}=0$ follows by a similar argument.

\subsection{Proof of Theorem 2.4.}

Proof. First we prove that the compatibility (2.6) is achieved.

$$
\left\|v_{0 \varepsilon}-\frac{\beta}{\lambda}\left|u_{0 \varepsilon}\right|^{2}\right\|_{L^{2}} \leq\left\|v_{0 \varepsilon}-\frac{\beta}{\lambda}\left|u_{0}\right|^{2}\right\|_{L^{2}}+\left\|\frac{\beta}{\lambda}\left|u_{0}\right|^{2}-\frac{\beta}{\lambda}\left|u_{0 \varepsilon}\right|^{2}\right\|_{L^{2}}
$$

and

$$
\begin{aligned}
\left\|\frac{\beta}{\lambda}\left|u_{0}\right|^{2}-\frac{\beta}{\lambda}\left|u_{0 \varepsilon}\right|^{2}\right\|_{L^{2}} & =\left|\frac{\beta}{\lambda}\right|||\left(\left|u_{0}\right|-\left|u_{0 \varepsilon}\right|\right)\left(\left|u_{0}\right|+\left|u_{0 \varepsilon}\right|\right) \|_{L^{2}} \\
& \lesssim\left\|\left|u_{0}\right|-\left|u_{0 \varepsilon}\right|\right\|_{L^{2}} \\
& \leq\left\|u_{0}-u_{0 \varepsilon}\right\|_{L^{2}},
\end{aligned}
$$

so

$$
\lim _{\varepsilon \rightarrow 0}\left\|v_{0 \varepsilon}-\frac{\beta}{\lambda}\left|u_{0 \varepsilon}\right|^{2}\right\|_{L^{2}}=0
$$


The same argument gives us that

$$
\begin{aligned}
\left\|v_{\varepsilon}-\frac{\beta}{\lambda}|u|^{2}\right\|_{L^{2}} & \leq\left\|v_{\varepsilon}-\frac{\beta}{\lambda}\left|u_{\varepsilon}\right|^{2}\right\|_{L^{2}}+\left|\frac{\beta}{\lambda}\right|\left\|\left|u_{\varepsilon}\right|^{2}-|u|^{2}\right\|_{L^{2}} \\
& \lesssim\left\|v_{\varepsilon}-\frac{\beta}{\lambda}\left|u_{\varepsilon}\right|^{2}\right\|_{L^{2}}+\left\|u_{\varepsilon}-u\right\|_{L^{2}},
\end{aligned}
$$

then

$$
\lim _{\varepsilon \rightarrow 0}\left\|v_{\varepsilon}-\frac{\beta}{\lambda}|u|^{2}\right\|_{L^{2}}=0
$$

Now let's check the $H^{1}$-convergence. First let's notice that as in the previous proof

$$
\left\|\partial_{x} \int_{0}^{t} S(t-s)\left[u_{\varepsilon} v_{\varepsilon}-\frac{\beta}{\lambda} u_{\varepsilon}\left|u_{\varepsilon}\right|^{2}\right](x, s) d s\right\|_{L_{T}^{\infty} L_{x}^{2}} \lesssim\left\|\partial_{x}\left[u_{\varepsilon}\left(v_{\varepsilon}-\frac{\beta}{\lambda}\left|u_{\varepsilon}\right|^{2}\right)\right]\right\|_{L_{T}^{4 / 3} L_{x}^{1}}
$$

and

$$
\begin{aligned}
\left\|\partial_{x}\left[u_{\varepsilon}\left(v_{\varepsilon}-\frac{\beta}{\lambda}\left|u_{\varepsilon}\right|^{2}\right)\right]\right\|_{L_{T}^{4 / 3} L_{x}^{1}} & \leq\left\|\partial_{x} u_{\varepsilon}\left(v_{\varepsilon}-\frac{\beta}{\lambda}\left|u_{\varepsilon}\right|^{2}\right)\right\|_{L_{T}^{4 / 3} L_{x}^{1}}+\left\|u_{\varepsilon} \partial_{x}\left(v_{\varepsilon}-\frac{\beta}{\lambda}\left|u_{\varepsilon}\right|^{2}\right)\right\|_{L_{T}^{4 / 3} L_{x}^{1}} \\
& \lesssim T^{3 / 4}\left(\left\|v_{\varepsilon}-\frac{\beta}{\lambda}\left|u_{\varepsilon}\right|^{2}\right\|_{L_{T}^{\infty} L_{x}^{2}}+\left\|\partial_{x}\left(v_{\varepsilon}-\frac{\beta}{\lambda}\left|u_{\varepsilon}\right|^{2}\right)\right\|_{L_{T}^{\infty} L_{x}^{2}}\right),
\end{aligned}
$$

so we need to look for $\partial_{x} w_{\varepsilon}$ as in the proof of Theorem 2.2 .

$$
\partial_{x} w_{\varepsilon}(x, t)=\partial_{x} w_{0 \varepsilon}\left(x-\frac{\lambda}{\epsilon} t\right)+\frac{\varepsilon \beta}{\lambda^{2}}\left[1-\mathcal{T}_{\varepsilon}(t)\right] \partial_{x}\left|u_{\varepsilon}(x, t)\right|^{2},
$$

then

$$
\left\|\partial_{x} w_{\varepsilon}(\cdot, t)\right\|_{L^{2}}=O(\varepsilon)
$$

and therefore

$$
\left\|\partial_{x} \int_{0}^{t} S(t-s)\left[u_{\varepsilon} v_{\varepsilon}-\frac{\beta}{\lambda} u_{\varepsilon}\left|u_{\varepsilon}\right|^{2}\right](x, s) d s\right\|_{L_{T}^{\infty} L_{x}^{2}}=T^{3 / 4} O(\varepsilon) .
$$

Now, this means that

$$
\left\|\partial_{x}\left(u_{\varepsilon}-S(t) u_{0 \epsilon}+i \frac{\alpha \beta}{\lambda} \int_{0}^{t} S(t-s) u_{\varepsilon}\left|u_{\varepsilon}\right|^{2} d s\right)\right\|_{L_{T}^{\infty} L_{x}^{2}} \lesssim T^{3 / 4} O(\varepsilon)
$$

and the proof is finished.

\subsection{Proof of Theorem 2.1.}

Proof. This theorem is a clear consequence of the procedure used to obtain the before results in the context of the Benney system (1.11).

\section{FinAl REMARKS}

Remark 6.1 (Local well-posedness). Notice that if it is considered $|\lambda|=\left|\lambda^{\prime}\right|=1$ in system (1.1), the same theory developed in [15] allows to conclude local well-posedness for this system for initial data $\left(u_{0}, v_{0}, z_{0}\right) \in H^{s} \times H^{\kappa} \times H^{\kappa}$ with $(s, \kappa)$ satisfying the conditions in (3.7). This fact follows immediately observing that the linear parts corresponding to the transport equations are not coupled.

Remark 6.2 (Conservation laws and global solutions). Formally, system (1.1) satisfies the following conservation laws:

$$
\begin{gathered}
\widetilde{\mathcal{M}}(t):=\int_{-\infty}^{+\infty}|u|^{2} d x=\widetilde{\mathcal{M}}(0) \quad(\text { mass }) \\
\widetilde{\mathcal{K}}(t):=\int_{-\infty}^{+\infty}\left[\frac{\alpha}{2 \beta} v^{2}+\frac{\alpha^{\prime}}{2 \beta^{\prime}} z^{2}+\frac{1}{\varepsilon} \operatorname{Im} u \bar{u}_{x}\right] d x=\widetilde{\mathcal{K}}(0) \quad(\text { moment })
\end{gathered}
$$

and

$$
\widetilde{\mathcal{E}}(t):=\int_{-\infty}^{+\infty}\left(\left|u_{x}\right|^{2}+\frac{\tau}{2}|u|^{4}+\alpha v|u|^{2}+\alpha^{\prime} z|u|^{2}-\frac{\alpha \lambda}{2 \beta} v^{2}-\frac{\alpha^{\prime} \lambda^{\prime}}{2 \beta^{\prime}} z^{2}\right) d x=\widetilde{\mathcal{E}}(0) \quad(\text { energy })
$$


with $\beta, \beta^{\prime} \neq 0$ and for all $0 \leq t<T_{\varepsilon}^{*}$, where $T_{\varepsilon}^{*}$ is the maximal time of existence for the respective solution.

Since the region of regularity in described in Remark 6.1 includes the case $H^{1} \times L^{2} \times L^{2}$ when $|\lambda|=\left|\lambda^{\prime}\right|=1$, the conservation laws above ensure global well-posedness in this case.

Remark 6.3. We observe that the same process used to prove Lemma 4.1 ensure that the condition (2.1) in Theorem 2.1 is valid in the case:

$$
\frac{\alpha \lambda}{\beta}<0 \quad \text { and } \quad \frac{\alpha^{\prime} \lambda^{\prime}}{\beta^{\prime}}<0
$$

We are currently adapting the ideas used in this work to obtain more accurate results in the same direction for the Zakharov system (1.5) in higher dimensions.

\section{REFERENCES}

[1] H. Added and S. Added; Equations of Langmuir Turbulence and Nonlinear Schrödinger Equation: Smoothness and Approximation. Journal of Functional Analysis 79, 183-21 (1988).

[2] J. Angulo, A. J. Corcho and S. Hakkaev; Well Posedness and Stability in the Periodic Case for the Benney system. Advances in Differential Equations 16, 523-550 (2011).

[3] I. Bejenaru, S. Herr, J. Holmer and D. Tataru; On the 2D Zakharov system with $L^{2}$ Schrödinger data, Nonlinearity, 22, 1063-1089 (2009).

[4] D. Bekiranov, T. Ogawa and G. Ponce; On the well-posedness of Benney's interaction equation of short and long waves. Advances in Differential Equations, 1, 919-937 (1996).

[5] D. Bekiranov, T. Ogawa and G. Ponce; Interaction equation for short and long dispersive waves. Journal of Functional Analysis, 158, 357-388 (1998).

[6] D. J. Benney; Significant interactions between small and large scale surface waves. Stud. Appl. Math., 55, 93-106 (1976).

[7] D. J. Benney; A general theory for interactions between short and long waves. Studies in Applied Math., 56, 81-94 (1977).

[8] D. Benney and G. Roskes; Waves instability. Studies in Applied Math., 48, 455-472 (1969).

[9] J. Bourgain and J. Colliander; On wellposedness of the Zakharov system. Int. Math. Res. Not., 11, 515-546 (1996).

[10] S. Champeaux, D. Laveder, T. Passot and P.L. Sulem; Remarks on the parallel propagation of small amplitude dispersive Alfven waves. Nonlinear Process. Geophys, 6, 169-178 (1999).

[11] A. J. Corcho; Ill-Posedness for the Benney system. Discrete and Continuous Dynamical Systems, 15, $965-972$ (2006).

[12] J. C. Cordero Ceballos; Supersonic limit for the Zakahrov-Rubenchik system. Journal of Differential Equations, 261, 5260-5288 (2016).

[13] Juan C. Cordero and José R. Quintero; Instability of the standing waves for a Benney-Roskes/Zakharov-Rubenchik system and blow-up for the Zakharov equations. Discrete and Continuous Dynamical Systems - B, 25 (4), 1213-1240 (2020).

[14] T. Cazenave; Semilinear Schrödinger equations. Courant Lecture Notes in Mathematics, vol. 10, New York: New York University Courant Institute of Mathematical Sciences, AMS (2003)

[15] J. Ginibre, Y. Tsutsumi and G. Velo; On the Cauchy Problem for the Zakharov system. Journal of Functional Analysis, 151, 384-436 (1997).

[16] C. Kenig, G. Ponce, and L. Vega; On the Zakharov and Zakharov-Schulman system. Journal of Functional Analysis, 127 (1), 204-234 (1995).

[17] E. Kuznetsov and V. Zakharov; Hamiltonian formalism for systems of hydrodinamics type. Mathematical Physics Reviews, Soviet Scientific Reviews, Section C, S. P. Novikov editor, 4, 167-220 (1984).

[18] Ph. Laurençot; On a nonlinear Schrödinger equation arising in the theory of water waves. Nonlinear Anal. TMA., 24, 509-527 (1995).

[19] F. Linares and C. Matheus; Well posedness for the Zakharov-Rubenchik system. Advances in Differential Equations, 14 (3-4), 261-288 (2009).

[20] F. Linares and G. Ponce; Introduction to Nonlinear Dispersive Equations. Unversitext - Springer (2009). 
[21] H. Luong, N. Mauser and J. Saut; On the Cauchy problem for the Zakharov-Rubenchik/Benney-Roskes system. Communications on Pure and Applied Analysis , 17 (4), 1573-1594 (2018).

[22] N. Masmoudi and K. Nakanishi; Energy convergence for singular limits of Zakharov type systems. Inventiones Mathematicae, 172, 535-583 (2008).

[23] F. Oliveira; Stability of the solitons for the one-dimensional Zakharov-Rubenchik equation. Physica D, 175, 220-240 (2003).

[24] F. Oliveira; Adiabatic limit of the Zakharov-Rubenchik equation. Reports on Mathematical Physics, 61 (1), 13-27 (2008).

[25] T. Ozawa and Y. Tsutsumi; The nonlinear Schrödinger limit and the initial layer of the Zakharov equations. Differential Integral Equations, 5, 721-745 (1992).

[26] H. Pecher; Global well-posedness below energy space for the 1-dimensional Zakharov system. Int. Math. Res. Not. no. (41), 1027-1056 (2001).

[27] H. Pecher; Global solutions with infinite energy for the one-dimensional Zakharov system. Electronic Journal of Differential Equations, 2005 (41), 1-18 (2005).

[28] G. Ponce and J. Saut; Well-posedness for the Benney-Roskes/Zakharov-Rubenchik system. Discrete and Continuous Dynamical Systema, 13, 811-825 (2005).

[29] A. Rubenchik and V. Zakharov; Nonlinear interaction of high-frequency and low-frequency waves. Prikl. Mat. Techn. Phys., 5, 84-98 (1972).

[30] S. Schochet and M. Weinstein; The nonlinear Schrödinger limit of the Zakharov equations governing Langmuir turbulence. Communications in Mathematical Physics, 106, 569-580 (1986).

[31] C. Sulem and P.L. Sulem; Quelques résultats de régularité pour les équations de la turbulence de langmuir. C.R. Acad. Sci. Paris, 289, 173-176 (1979).

[32] C. Sulem and P.L. Sulem; The Nonlinear Schrödinger Equation: Self-Focusing and Wave Collapse. Applied Mathematical Sciences, Springer-Verlag New York, Inc., 139 (1999)

[33] M. Tsutsumi and S. Hatano; Well-posedness of the Cauchy Problem for long wave - short wave resonance equation. Nonlinear Anal. TMA., 22, 155-171 (1994).

[34] M. Tsutsumi and S. Hatano; Well-posedness of the Cauchy Problem for Benney's firsts equations of long wave short wave interactions. Funkcialaj Ekvacioj, 37, 289-316 (1994).

[35] V. Zakharov; Collapse of langmuir waves. Sov. Phys. JETP, 35, 908-914 (1972). 